\title{
Clinical Impact of Escalating Relative High-dose-rate Intracavitary Brachytherapy Dose in Stage IIB Cervical Cancer
}

\author{
OYEON CHO ${ }^{1}, \mathrm{O} \mathrm{KYU} \mathrm{NOH}^{1}$, YOUNG-TAEK OH ${ }^{1}$, SUK-JOON CHANG ${ }^{2}$ and MISON CHUN ${ }^{1}$ \\ Departments of ${ }^{1}$ Radiation Oncology and ${ }^{2}$ Obstetrics and Gynecology, \\ Ajou University School of Medicine, Suwon, Republic of Korea
}

\begin{abstract}
Background/Aim: To investigate whether highdose-rate (HDR) intracavitary brachytherapy (IBT) dose ratios can predict treatment outcomes in patients with stage IIB cervical cancer. Patients and Methods: Ninety-three patients treated with weekly cisplatin-based concurrent chemoradiotherapy and HDR IBT were analyzed. Potential prognostic factors and treatment outcomes were compared between low-HDR-IBT-ratio $(\leq 0.43)$ and high-HDR-IBTratio (>0.43) groups, and univariate and multivariate analyses were performed. Results: Five-year disease-specific survival (DSS) and progression-free survival (PFS) rates were significantly shorter in the low-compared to the highHDR-IBT-ratio group. A high HDR IBT ratio was confirmed as an independent prognostic factor for DSS and PFS. Conclusion: A high HDR IBT dose ratio improves DSS and PFS in patients with stage IIB cervical cancer. Therefore, active administration of HDR IBT beyond previously accepted levels may be necessary for the treatment of locally advanced cervical cancer.
\end{abstract}

The current treatment for patients with locally advanced cervical cancer is weekly cisplatin-based concurrent chemoradiotherapy (CCRT) followed by intracavitary brachytherapy (IBT) (1). In particular, IBT in combination with central pelvic external-beam radiotherapy (EBRT) has played a key role in increasing survival in patients with locally advanced cervical cancer.

A previous study demonstrated that the use of active IBT with a central pelvic EBRT dose (CPD) of 40.0-45.0 Gy resulted in favourable treatment outcomes with low complication rates in patients with International Federation

Correspondence to: Mison Chun Department of Radiation Oncology, Ajou University School of Medicine, 206 World cup-ro, Yeongtong-gu, Suwon 16499, Korea. Tel: +82 0312195884, Fax: +82 0312195894, e-mail: chunm@ajou.ac.kr

Key Words: Cervical cancer, high-dose-rate intracavitary brachytherapy, survival. of Gynaecology and Obstetrics (FIGO) stage IIIB cervical cancer (2). In an American population-based study, treatment of patients with FIGO stage IB-IVA disease with IBT and central pelvic EBRT was associated with a better survival rate than treatment with central pelvic EBRT alone (3). This was no different in patients who had received high-dose-rate (HDR) IBT to replace low-dose-rate IBT. A 2012 report of international brachytherapy patterns for cervical cancer revealed that a Japanese group used a total dose of $65.7 \mathrm{~Gy}$ [equivalent dose in 2.0 Gy fractions (EQD2) using an alpha/beta ratio of $10.0 \mathrm{~Gy}$ at point A] (4), which is significantly lower than the 80.9 Gy (EQD2) reported in other countries. Conversely, the mean HDR IBT ratio, defined as the ratio of the HDR IBT dose (EQD2) to the sum of the CPD and HDR IBT dose (EQD2), was reported to be 0.51 in Japan, which was significantly higher than the average of all other countries (0.45) (4). Japanese trials that have been conducted using both a low total EQD2 at point A (52.0 Gy for FIGO stage I-II, and 62.0 Gy for FIGO stage III-IVA) and a high HDR IBT ratio ( 0.61 for FIGO stage I-II, and 0.51 for FIGO stage III-IVA) achieved treatment outcomes that are comparable to those using conventional treatment methods $(5,6)$. Therefore, it may be beneficial to increase the HDR IBT ratio for the treatment of primary tumours.

Despite indirect evidence of the clinical significance of the HDR IBT ratio, there have been no immediate studies addressing it. Therefore, we aimed to investigate whether the HDR IBT ratio is associated with treatment outcomes in patients with FIGO stage IIB cervical cancer.

\section{Patients and Methods}

Study population. This study was approved by the Institutional Review Board (AJIRB-MED-OBS-16-031) of Ajou University Hospital (Suwon, Republic of Korea). All study participants provided written informed consent. Research was conducted in accordance with the 1964 Declaration of Helsinki and the Title 45, U.S. Code of Federal Regulations, Part 46, Protection of Human Subjects (effective 13th December 2001).

In total, 93 patients who were clinically diagnosed with FIGO stage IIB cervical cancer and treated with weekly cisplatin-based 
pelvic CCRT and HDR IBT between August 2001 and November 2013 were selected. All patients had undergone a physical examination and tumour histology was confirmed by a uterine cervical biopsy. Squamous cell carcinoma (SCC) antigen level, complete blood counts, and imaging data, including chest radiography, pelvic magnetic resonance imaging (MRI), with/without positron-emission tomography-computed tomography, were acquired at diagnosis. Cystoscopy and sigmoidoscopy were performed in patients with suspected rectal or bladder invasion. These patients were followed-up every 3 months for the first 3 years and every 6 months thereafter. The median follow-up of the whole patient group was 59 (range $=15-136)$ months. Disease progression was evaluated by physical examination, Pap smear, MRI, or positron-emission tomography-computed tomography. Patients with disease progression were treated with chemotherapy, radiotherapy, or conservative care. The endpoints of this study were diseasespecific survival (DSS) and progression-free survival (PFS). Radiation-related toxicities that occurred 8 months after the end of treatment were regarded as late complications.

Radiotherapy and chemotherapy. EBRT was delivered with a 10.015.0 MV photon beam to the whole pelvis until a total dose of $45.0 \mathrm{~Gy}$ ( 25 fractions of $1.8 \mathrm{~Gy}$ per day for 5 weeks) was achieved. Thirty-one patients underwent dose modification, including 18.0 Gy delivered in 12 fractions or 15.0 Gy delivered in 10 fractions twice daily in the third week, in order to shorten the overall treatment duration (defined as the time interval between the commencement and completion of radiotherapy) (7). These patients were treated to a total dose of 45.0 or $45.6 \mathrm{~Gy}$, delivered in 27 fractions. The parametrium and lymph nodes were boosted with 3-5 fractions of 5.4-10.0 Gy after whole-pelvic EBRT. Midline blocks $(4.0 \mathrm{~cm}$ in width) were applied after 36.0-51.5 Gy in 87 patients; inferior and superior borders were below the radiation field and above the tandem insertion site, respectively. Midline blocks were not applied in the remaining six patients. Twodimensional HDR IBT with iridium-192 (MicroSelectron; Nucletron Technologies GmbH, Munich, Germany) was implemented biweekly after midline block insertion. The prescribed dose was 10.0-30.0 Gy (2-7 fractions of 4.0-5.0 Gy per fraction) at point A. Its location, as defined by the International Commission on Radiation Units and Measurements (Report 38), was modified to 1.0 or $1.5 \mathrm{~cm}$ in patients with a small uterus. The EQD2 using an alpha/beta ratio of $3.0 \mathrm{~Gy}$ for the bladder and rectal points was planned to be delivered to $<80.0 \mathrm{~Gy}$ or $<70.0 \mathrm{~Gy}$ in twodimensional simulations, respectively. The total dose (EQD2) was defined as the sum of the CPD and the HDR IBT dose (EQD2) at point A. The HDR IBT ratio was defined as the ratio of the HDR IBT dose (EQD2) to the total dose (EQD2). Between 40.0 and $70.0 \mathrm{mg} / \mathrm{m}^{2}$ of cisplatin was administered concurrently with central pelvic EBRT for 4-6 consecutive weeks. Chemotherapy was delayed for patients with absolute neutrophil counts of $<500 \mathrm{cells} / \mu \mathrm{l}$ or platelet counts of $<50,000 \mathrm{cells} / \mu \mathrm{l}$. Granulocyte colony-stimulating factor and intravenous antibiotics were administered to patients with neutropenic fever.

Measurements of volumetric parameters. Of the 93 patients who underwent pre-treatment pelvic MRI, imaging data was available for 89 patients. Tumour areas were defined using the Eclipse ${ }^{\text {TM }}$ Treatment Planning System, version 10.0 (Varian Medical Systems, Palo Alto, CA, USA). From the initial MRI, we were able to determine whether the cervical tumour had deviated in the anterior (bladder) or posterior (rectum) direction or not. Forty-two patients underwent pelvic MRI at 36.0-45.0 Gy. The tumour volumes of these patients were delineated in the same manner. The residual tumour volume was defined as the pre-treatment tumour volume (V1) divided by the tumour volume at 36.0-45.0 Gy (V2).

Statistical analyses. Initially, we analysed volumetric factors and fluctuations in the baseline CPD that might be relevant to the determination of CPD followed by the HDR IBT dose (EQD2). Next, we compared potential prognostic factors [age, pathological subtype, tumour size, pelvic lymph node involvement, SCC antigen/haemoglobin levels, overall treatment duration, and total dose (EQD2)] and treatment outcomes between patients with a low HDR IBT ratio and patients with a high HDR IBT ratio. Fisher's exact tests or chi-square tests were performed for categorical variables and $t$-tests or Mann-Whitney $U$-tests were performed for continuous variables. SCC antigen levels were categorised according to their median value. All potential prognostic factors, including the HDR IBT ratio, were analysed using the KaplanMeier method or a Cox proportional hazards model. The Cox proportional hazards model was used for multivariate analysis, including factors with a $p<0.10$ from the univariate analysis. All statistical analyses were performed using $\mathrm{R}$ software version 3.2.3 (The R Foundation for Statistical Computing, Vienna, Austria). A value of $p<0.05$ was considered statistically significant.

\section{Results}

Patient characteristics. A summary of the patients' demographic and clinicopathological characteristics, and volumetric parameters are provided in Table I.

Treatment outcomes. Seventeen out of 93 patients experienced disease progression. Two patients experienced relapse at the cervix without distant metastases (DMs). The residual cervical tumour progressed after treatment in four patients with DMs and in the remaining 11 patients, DMs occurred without local progression. Metastatic sites included the para-aortic lymph nodes ( $n=4$ patients), lungs $(n=3$ patients), bone ( $n=3$ patients), peritoneum ( $n=2$ patients), liver ( $\mathrm{n}=2$ patients), back muscles $(\mathrm{n}=1$ patient), supraclavicular lymph nodes $(\mathrm{n}=1$ patient $)$, and adrenal gland $(n=1$ patient). Of the 17 patients with disease progression, nine received $>3$ cycles of second-line chemotherapy (cisplatin plus paclitaxel). The remaining eight patients received conservative treatment because they were either intolerant to or had refused chemotherapy. Fourteen patients died of cervical cancer. The 5-year DSS and PFS rates for all patients combined were $85.9 \%$ and $81.6 \%$, respectively.

Central pelvic EBRT doses. Figure 1 indicates that the baseline CPD increased from 35.4 to 44.3 Gy (EQD2) with increasing numbers of patients undergoing pelvic MRI during CCRT from 2007. The CPD (mean \pm standard deviation) was significantly higher in patients who had undergone pelvic 
Table I. Patient demographic and clinicopathological characteristics.

\begin{tabular}{|c|c|}
\hline Characteristic & Value \\
\hline \multicolumn{2}{|l|}{ Age at diagnosis } \\
\hline Median (range), years & $56(30-82)$ \\
\hline$\leq 65$ Years, $\mathrm{n}(\%)$ & $59(63.4)$ \\
\hline$>65$ Years, $\mathrm{n}(\%)$ & $34(36.6)$ \\
\hline \multicolumn{2}{|l|}{ Histology, n (\%) } \\
\hline ADSC/ADC & $5(5.4)$ \\
\hline SCC & 88 (94.6) \\
\hline \multicolumn{2}{|c|}{ Pelvic lymph node involvement, n (\%) } \\
\hline Present & $62(66.7)$ \\
\hline Absent & $31(33.3)$ \\
\hline \multicolumn{2}{|l|}{ SCC antigen level } \\
\hline Median (range), $\mathrm{ng} / \mathrm{ml}$ & $4.4(0.5-59.4)$ \\
\hline \multicolumn{2}{|l|}{ Haemoglobin level } \\
\hline Median (range), g/dl & $11.7(4.9-13.0)$ \\
\hline$\leq 11 \mathrm{~g} / \mathrm{dl}, \mathrm{n}(\%)$ & $25(26.9)$ \\
\hline$>11 \mathrm{~g} / \mathrm{dl}, \mathrm{n}(\%)$ & $68(73.1)$ \\
\hline \multicolumn{2}{|l|}{ Overall treatment duration } \\
\hline Median (range), days & $51(41-78)$ \\
\hline$\leq 56$ Days, $\mathrm{n}(\%)$ & $71(76.3)$ \\
\hline$>56$ Days, $\mathrm{n}(\%)$ & $22(23.7)$ \\
\hline \multicolumn{2}{|l|}{ CPD (EQD2), Gy } \\
\hline Median (range) & $40.1(35.4-59.4)$ \\
\hline \multicolumn{2}{|l|}{ HDR IBT dose (EQD2) } \\
\hline Median (range), Gy & $31.3(12.5-37.5)$ \\
\hline \multicolumn{2}{|l|}{ Total dose (EQD2)a } \\
\hline Median (range), Gy & $68.1(59.0-87.1)$ \\
\hline$\leq 70$ Gy & $52(55.9)$ \\
\hline$>70 \mathrm{~Gy}$ & $41(44.1)$ \\
\hline \multicolumn{2}{|l|}{ HDR IBT ratio ${ }^{b}$} \\
\hline Median (range) & $0.43(0.18-0.48)$ \\
\hline \multicolumn{2}{|l|}{ Tumour volume on MRIc } \\
\hline \multicolumn{2}{|c|}{ Pre-treatment tumour volume (V1) } \\
\hline Median (range), cc & $37(3.1-164.2)$ \\
\hline$\leq 50 \mathrm{cc}, \mathrm{n}(\%)$ & $59(66.3)$ \\
\hline$>50 \mathrm{cc}, \mathrm{n}(\%)$ & $30(33.7)$ \\
\hline \multicolumn{2}{|c|}{ Tumour volume at $36-45$ Gy (V2) } \\
\hline Median (range), cc & $4.5(0.0-38.2)$ \\
\hline$\leq 10 \mathrm{cc}, \mathrm{n}(\%)$ & $35(83.3)$ \\
\hline$>10 \mathrm{cc}, \mathrm{n}(\%)$ & $7(16.7)$ \\
\hline \multicolumn{2}{|l|}{$\mathrm{V} 1+\mathrm{V} 2$} \\
\hline Median (range), cc & $37.2(3.1-140.5)$ \\
\hline \multicolumn{2}{|l|}{ V1 only } \\
\hline Median (range), cc & $36.5(4.4-164.2)$ \\
\hline \multicolumn{2}{|c|}{ Residual tumour volume (V1/V2) } \\
\hline Median (range), $\%$ & $14.2(0.0-52.4)$ \\
\hline$\leq 20 \%, \mathrm{n}(\%)$ & $32(76.2)$ \\
\hline$>20 \%, \mathrm{n}(\%)$ & $10(23.8)$ \\
\hline \multicolumn{2}{|l|}{ Tumour asymmetryc ${ }^{\mathrm{c}}, \mathrm{n}(\%)$} \\
\hline Anterior (bladder) & $12(13.5)$ \\
\hline Posterior (rectum) & $20(22.5)$ \\
\hline None & $57(64.0)$ \\
\hline
\end{tabular}

ADC, Adenocarcinoma; ADSC, adenosquamous carcinoma; CPD, central pelvic external-beam radiotherapy dose; EQD2, equivalent dose in 2-Gy fractions using an alpha/beta ratio of $10 \mathrm{~Gy}$ at point A; HDR, high-dose rate; IBT, intracavitary brachytherapy; MRI: magnetic resonance imaging; SCC, squamous cell carcinoma. ${ }^{\text {aDefined }}$ as the sum of the central pelvic EBRT dose (EQD2) and the HDR IBT dose (EQD2); bdefined as the ratio of the HDR IBT dose to the total dose (EQD2); cimaging data were not available for four out of 93 patients who underwent pre-treatment magnetic resonance imaging.
MRI during CCRT compared to patients who had not undergone pelvic MRI during CCRT [38.3 \pm 3.9 vs. $45.9 \pm 5.6$ Gy (EQD2); $p<0.001]$. Table II suggests that the determination of CPDs for patients who had not undergone pelvic MRI during CCRT was related to tumor symmetry and a large V1, whereas the determination of CPDs for patients who had undergone MRI during CCRT was related to a large V2. However, the HDR IBT ratio was lower in the group with the asymmetric tumor and V1 $>50.0 \mathrm{cc}$ than the other three groups with symmetric tumor and V1 $\leq 50.0 \mathrm{cc}$, asymmetric and V1 $\leq 50.0 \mathrm{cc}$, or symmetric tumor and V1 $>50.0 \mathrm{cc}$. Conversely, HDR IBT ratios were not significantly different between the V2 $\leq 10.0 \mathrm{cc}$ and V2 $>10.0$ cc groups.

Clinical findings according to HDR IBT ratio. Table III indicates that 13 patients in the low-HDR-IBT-ratio group $(\leq 0.43)$ developed disease progression, including two with local recurrence, seven with DMs, and four with both local recurrence and DMs. Eleven out of these 13 patients experienced disease-related death. Conversely, three out of four patients with DMs in the group with high HDR IBT ratio $(>0.43)$ experienced disease-related death $(p=0.020$ and $p=0.026$, respectively). The 5-year DSS and PFS rates were significantly lower in the group with low HDR IBT ratio compared to the group with the high HDR IBT ratio (5-year DSS: $78.3 \%$ vs. $93.1 \% ; p=0.003$ and 5-year PFS: $72.7 \%$ vs. $90.7 \% ; p=0.007$, respectively). Kaplan-Meier curves comparing treatment outcomes between the two groups (stratified according to the median HDR IBT ratio) highlight these differences (Figure 2A-B). Two out of four patients with confirmed rectal bleeding from sigmoidoscopy [total dose, 77.2 and 49.2 Gy (EQD2) and CPD, 75.5 and 44.3 Gy (EQD2), respectively] were treated with coagulation. For the remaining two patients, this complication was self-limited [total dose, 68.1 and 35.4 Gy (EQD2) and CPD, 71.6 and 38.9 Gy (EQD2), respectively]. One patient developed a radiation-induced vesicovaginal fistula, confirmed by an urologist and treated with an iliac conduit [total dose, 80.1 Gy (EQD2) and CPD, 56.7 Gy (EQD2)]. The group with low HDR IBT ratio included three patients who required intervention for late complications, whereas that with high HDR IBT ratio included two patients who did not require any intervention.

Multivariate analyses. Table IV summarises the univariate and multivariate analyses of all potential prognostic factors. Adjusted hazard ratios (HRs) for patients in the group with high HDR IBT ratio were independently low for both DSS $(\mathrm{HR}=0.26,95 \%$ confidence interval $=0.06-1.02 ; p=0.053)$ and PFS $(\mathrm{HR}=0.25,95 \%$ confidence interval=0.06-0.96; $p=0.043$ ). The group aged $>65$ years had an independently high adjusted $\mathrm{HR}$ for $\mathrm{DSS}(\mathrm{HR}=3.09,95 \%$ confidence interval=0.99-9.62; $p=0.052$ ). 


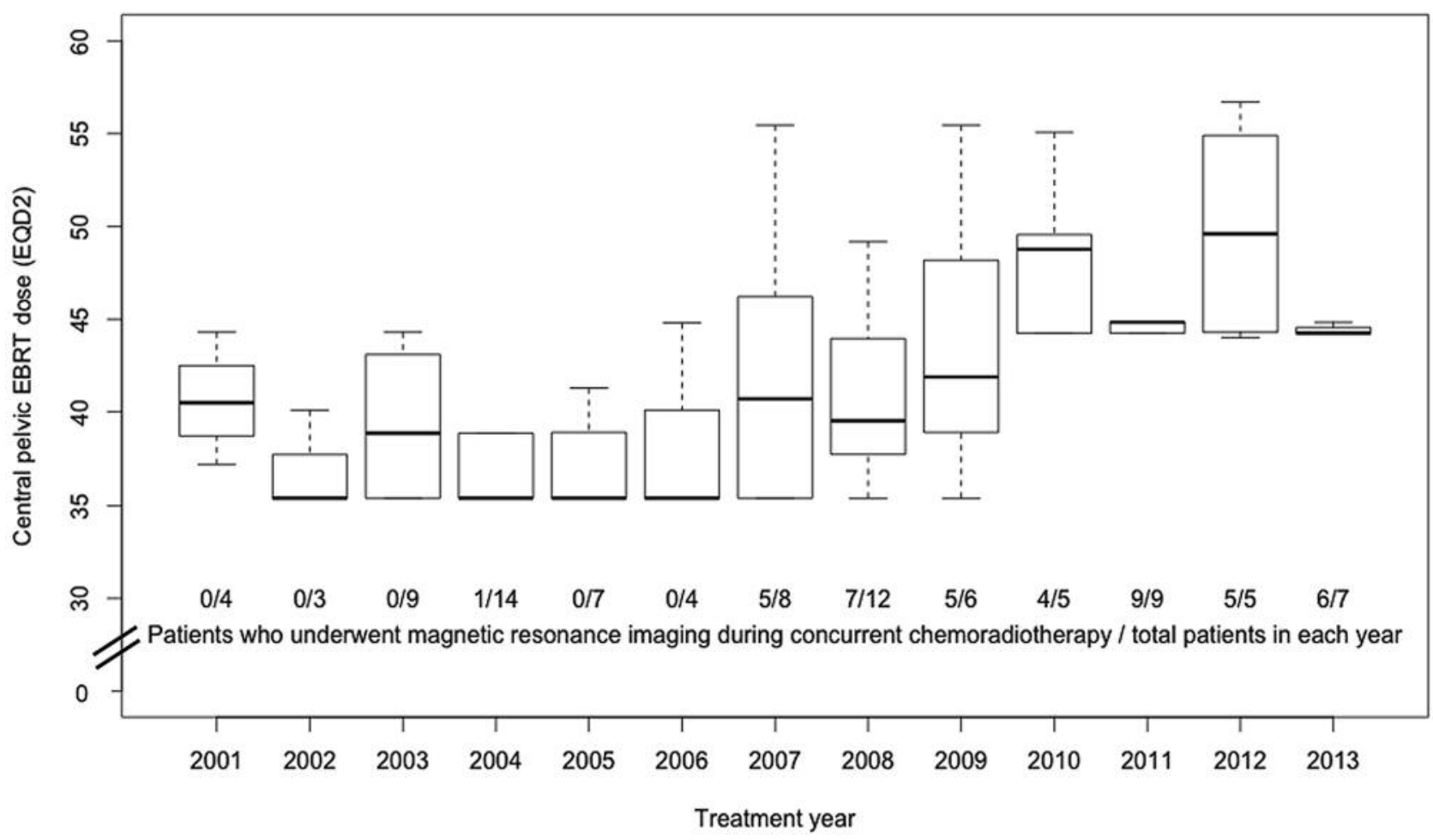

Figure 1. Relationships of baseline central pelvic external beam radiotherapy (EBRT) dose (EQD2, equivalent dose in 2-Gy fractions using an alpha/beta ratio of 10 Gy at point A with treatment years.

Table II. Associations between pre-treatment tumour volumes, tumour symmetry and residual tumour volumes and determination of central pelvic external-beam radiotherapy dose.

\begin{tabular}{|c|c|c|c|c|c|}
\hline Tumour volume on MRI, cc & $\mathrm{V} 1(\leq 50 \mathrm{cc})+\mathrm{SYM}$ & $\mathrm{V} 1(\leq 50 \mathrm{cc})+\mathrm{ASYM}$ & $\mathrm{V} 1(>50 \mathrm{cc})+\mathrm{SYM}$ & $\mathrm{V} 1(>50 \mathrm{cc})+\mathrm{ASYM}$ & $p$-Value \\
\hline V1 only $(n=47)$ & $(n=20)$ & $(\mathrm{n}=11)$ & $(n=11)$ & $(\mathrm{n}=5)$ & - \\
\hline CPD (EQD2), Gy & $37.7 \pm 3.8$ & $37.3 \pm 2.8$ & $37.8 \pm 3.1$ & $43.1 \pm 6.0$ & $0.048 *$ \\
\hline HDR IBT dose (EQD2), Gy & $31.1 \pm 2.6$ & $32.2 \pm 1.4$ & $32.2 \pm 1.4$ & $27.1 \pm 6.1$ & $0.023 *$ \\
\hline HDR IBT ratio & $0.45 \pm 0.04$ & $0.46 \pm 0.02$ & $0.46 \pm 0.03$ & $0.39 \pm 0.09$ & 0.072 \\
\hline$V 1+V 2(n=42)$ & $(n=16)$ & $(n=12)$ & $(\mathrm{n}=10)$ & $(n=4)$ & - \\
\hline CPD (EQD2), Gy & $44.6 \pm 6.0$ & $46.9 \pm 4.8$ & $46.4 \pm 5.7$ & $47.1 \pm 6.9$ & 0.261 \\
\hline HDR IBT dose (EQD2), Gy & $25.6 \pm 7.1$ & $28.0 \pm 5.9$ & $25.8 \pm 8.0$ & $25.7 \pm 9.8$ & 0.779 \\
\hline HDR IBT ratio & $0.36 \pm 0.09$ & $0.37 \pm 0.06$ & $0.35 \pm 0.09$ & $0.35 \pm 0.11$ & 0.819 \\
\hline & \multicolumn{2}{|c|}{$\mathrm{V} 2(\leq 10 \mathrm{cc})$} & \multicolumn{2}{|c|}{$\mathrm{V} 2(>10 \mathrm{cc})$} & \\
\hline$V 1+V 2(n=42)$ & \multicolumn{2}{|c|}{$(n=35)$} & \multicolumn{2}{|c|}{$(n=7)$} & - \\
\hline CPD (EQD2), Gy & \multicolumn{2}{|c|}{$45.2 \pm 5.8$} & \multicolumn{2}{|c|}{$49.2 \pm 3.3$} & 0.089 \\
\hline HDR IBT dose (EQD2), Gy & \multicolumn{2}{|c|}{$26.2 \pm 6.8$} & \multicolumn{2}{|c|}{$26.5 \pm 9.3$} & 0.923 \\
\hline HDR IBT ratio & \multicolumn{2}{|c|}{$0.37 \pm 0.08$} & \multicolumn{2}{|c|}{$0.34 \pm 0.09$} & 0.524 \\
\hline & \multicolumn{2}{|c|}{ Residual tumour volume (V1/V2) $(\leq 20 \%)$} & \multicolumn{2}{|c|}{ Residual tumour volume (V1/V2) (>20\%) } & \\
\hline $\mathrm{V} 1+\mathrm{V} 2(\mathrm{n}=42)$ & \multicolumn{2}{|c|}{$(n=32)$} & \multicolumn{2}{|c|}{$(\mathrm{n}=10)$} & - \\
\hline CPD (EQD2), Gy & \multicolumn{2}{|c|}{$45.1 \pm 5.4$} & \multicolumn{2}{|c|}{$48.5 \pm 5.9$} & 0.100 \\
\hline HDR IBT dose (EQD2), Gy & \multicolumn{2}{|c|}{$25.9 \pm 6.2$} & \multicolumn{2}{|c|}{$27.4 \pm 10.0$} & 0.649 \\
\hline HDR IBT ratio & \multicolumn{2}{|c|}{$0.37 \pm 0.08$} & \multicolumn{2}{|c|}{$0.34 \pm 0.09$} & 0.798 \\
\hline
\end{tabular}

ASYM, Tumour asymmetry; CPD, central pelvic external beam radiotherapy dose; EQD2, equivalent dose in 2-Gy fractions using an alpha/beta ratio of $10 \mathrm{~Gy}$ at point A; HDR, high-dose rate; IBT, intracavitary brachytherapy; MRI, magnetic resonance imaging; SYM, tumour symmetry; V1, pre-treatment tumour volume; V2, tumour volume at 36-45 Gy. Data are means \pm standard deviation. *Significantly different at $p<0.05$. 
Table III. Two-group comparisons according to the median high-dose-rate (HDR) intracavitary brachytherapy (IBT) ratio.

\begin{tabular}{|c|c|c|c|}
\hline \multirow[t]{2}{*}{ Factor } & \multicolumn{2}{|c|}{ HDR IBT ratio } & \multirow[t]{2}{*}{$p$-Value } \\
\hline & $\leq 0.43(n=47)$ & $>0.43(\mathrm{n}=46)$ & \\
\hline Age at diagnosis (>65 years), $\mathrm{n}(\%)$ & $23(48.9)$ & $11(23.9)$ & $0.022 *$ \\
\hline Histology (ADSC/ADC), n (\%) & $1(2.1)$ & $4(8.7)$ & 0.345 \\
\hline Pelvic lymph node involvement, $\mathrm{n}(\%)$ & $30(63.8)$ & $32(69.6)$ & 0.714 \\
\hline Haemoglobin level (>11 g/dl), n (\%) & $33(70.2)$ & $35(76.1)$ & 0.686 \\
\hline SCC antigen level $(>4.4 \mathrm{ng} / \mathrm{ml}), \mathrm{n}(\%)$ & $27(57.4)$ & $19(41.3)$ & 0.177 \\
\hline Overall treatment duration ( $>56$ days), $\mathrm{n}(\%)$ & $14(29.8)$ & $8(17.4)$ & 0.245 \\
\hline Use of MRI at $36-45 \mathrm{~Gy}, \mathrm{n}(\%)$ & $33(73.3)$ & $9(20.5)$ & $<0.001 *$ \\
\hline \multicolumn{4}{|l|}{ Total dose (EQD2) } \\
\hline$>70$ Gy, n (\%) & $25(53.2)$ & $16(34.8)$ & 0.114 \\
\hline Mean \pm SD & $70.6 \pm 5.1$ & $70.8 \pm 4.8$ & 0.865 \\
\hline CPD (EQD2) ${ }^{\mathrm{a}}, \mathrm{Gy}$ & $45.8 \pm 5.4$ & $37.6 \pm 3.2$ & $<0.001 *$ \\
\hline HDR IBT dose (EQD2)a ${ }^{\mathrm{a}}, \mathrm{Gy}$ & $24.8 \pm 5.4$ & $33.2 \pm 1.8$ & $<0.001 *$ \\
\hline Disease progression, $\mathrm{n}(\%)$ & $13(27.7)$ & $4(8.7)$ & $0.036^{*}$ \\
\hline Local progression & $2(4.3)$ & $0(0.0)$ & 0.484 \\
\hline Local progression + DM & $4(8.5)$ & $0(0.0)$ & 0.131 \\
\hline $\mathrm{DM}$ & $7(14.9)$ & $4(8.7)$ & 0.546 \\
\hline Disease-specific death, n (\%) & $11(23.4)$ & $3(6.5)$ & $0.047 *$ \\
\hline 5-year PFS rate, $\%$ & 72.7 & 90.7 & $0.007 *$ \\
\hline 5-year DSS rate, $\%$ & 78.3 & 93.1 & $0.003 *$ \\
\hline \multicolumn{4}{|l|}{ Late complications, $\mathrm{n}(\%)$} \\
\hline Rectal bleeding - self-limited & $0(0.0)$ & $2(4.3)$ & \\
\hline Rectal bleeding - coagulation & $2(4.3 \%)$ & $0(0.0)$ & \\
\hline Vesicovaginal fistula & $1(2.1)$ & $0(0.0)$ & 0.083 \\
\hline \multicolumn{4}{|l|}{ Tumour volumes on MRI ${ }^{\mathrm{b}}, \mathrm{n}(\%)$} \\
\hline Pre-treatment tumour volume (V1 >50 cc) & $17(37.8)$ & $13(29.5)$ & 0.555 \\
\hline Tumour asymmetry (anterior/posterior) & $17(37.8)$ & $15(34.1)$ & 0.887 \\
\hline
\end{tabular}

ADSC, Adenosquamous carcinoma; ADC, adenocarcinoma; CPD, central pelvic external-beam radiotherapy dose; DM, distant metastasis; DSS, disease-specific survival; EQD2, equivalent dose in 2-Gy fractions using an alpha/beta ratio of $10 \mathrm{~Gy}$ at point A; MRI, magnetic resonance imaging; PFS, progression-free survival; SCC, squamous cell carcinoma. ${ }^{\mathrm{a} M e a n} \pm$ standard deviation; bimaging data were not available for four out of 93 patients who underwent pre-treatment MRI. *Significantly different at $p<0.05$.

\section{Discussion}

HDR IBT ratios predicted treatment outcomes in patients with FIGO stage IIB cervical cancer treated with CCRT followed by HDR IBT. Patients in the group with low HDR IBT ratio had significantly shorter DSS and PFS rates compared to those of the group with high HDR IBT ratio, with no significant differences observed for conventional prognostic factors. Elevated HDR IBT ratios were associated with longer DSS and PFS rates.

There may have been more patients in the group with low HBR IBT ratio than in that with a high HBR IBT ratio who did not respond rapidly to central pelvic EBRT since the CPD may be determined by tumour response during central pelvic EBRT. Moreover, a large tumour volume and poor response to central pelvic EBRT (EQD2) might result in a low HDR IBT ratio. Therefore, it must be established whether the CPD was determined by tumour response or not before explaining the clinical significance of HDR IBT ratios. Our data suggest that patients who had undergone MRI at 36.0-45.0 Gy had a significantly higher baseline CPD compared to patients who had not. In particular, the CPD for patients who did not have MRI data available for tumour responses was higher in the group with both tumour asymmetry and a V1 of $>50.0$ cc compared to the other groups. This resulted in a low HDR IBT ratio. The increase in the CPD may be explained by concerns of insufficient dose coverage in the asymmetric region, regardless of tumour response. Conversely, patients who had undergone MRI during CCRT tended to have a higher CPD in the V2 $>10.0$ cc group compared to the $\mathrm{V} 2 \leq 10.0 \mathrm{cc}$ group. This did not result in a reduction in HDR IBT ratio due to maintaining the HDR IBT dose (EQD2). Finally, we were unable to find any evidence in support of an association between tumour response and HDT IBT ratios from our data.

The group with low HDR IBT ratio included a greater proportion of patients who had undergone MRI at 36.0-45.0 Gy and with an age of $>65$ years compared to the group with 


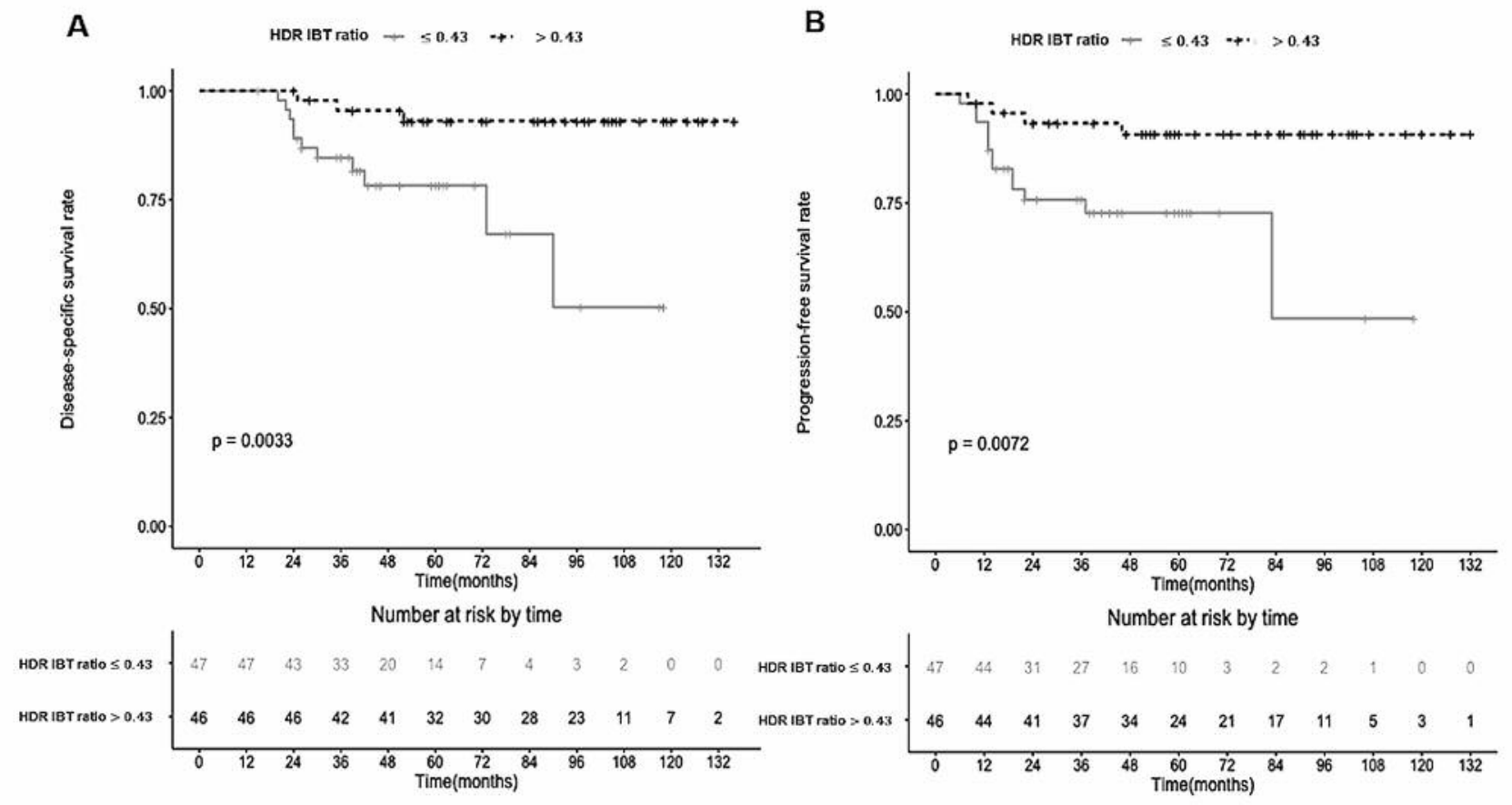

Figure 2. Kaplan-Meier plots of disease-specific (A) and progression-free (B) survival rates of patients with stage IIB cervical cancer stratified according to a median high-dose-rate (HDR) intracavitary brachytherapy (IBT) ratio of 0.43. Patients with a low ( $\leq 0.43)$ or a high $(>0.43) \mathrm{HDR}$ IBT ratio are represented by solid and dashed lines, respectively. The HDR IBT ratio was defined as the ratio of the HDR IBT dose (EQD2, equivalent dose in 2-Gy fractions using an alpha/beta ratio of $10 \mathrm{~Gy}$ at point $\mathrm{A}$ to the sum of the central pelvic external beam radiotherapy dose (EQD2) and the HDR IBT dose (EQD2).

high HDR IBT ratio. There was also no statistically significant relevance of total dose (EQD2) analyses. Variables such as pathology, pelvic lymph node involvement, SCC antigen or haemoglobin levels, overall treatment duration, total dose (EQD2), V1, and tumour asymmetry were not significant prognostic factors for DSS or PFS in patients with FIGO stage IIB cervical cancer. Their prognostic effects in matching FIGO stage patients may be attenuated although they were shown to be important for predicting survival in patients with locally advanced cervical cancer (8-10). This may have arisen from an association between FIGO stage and these variables, as well as the exclusion of patients who underwent radiotherapy field modification because of a bulky tumour, pelvic lymph node involvement, or high SCC antigen level. An older age of $>65$ years was a significant predictor of poorer DSS in the univariate analysis. However, this finding is in disagreement with a previous study (11), which revealed comparable treatment outcomes for elderly and nonelderly patients. This could be because the group with low HDR IBT ratio included more patients aged $>65$ years compared to that with high HDR IBT ratio. Moreover, six out of nine patients $(66.7 \%)$ who had received inadequate second-line chemotherapy after progression were aged $>65$ years, whilst two out of eight patients $(25.0 \%)$ who did receive adequate second-line chemotherapy after progression were aged $\leq 65$ years $(p>0.05)$.

Previous reports supported the notion that brachytherapy can improve the survival rate of patients with cervical cancer (2-4). Therefore, we hypothesised that elevated HDR IBT ratios might be relevant to improving treatment outcomes in these patients. This strategy might be effective for the prevention of DMs, as well as the suppression of local progression. If patients with both local progression and DMs are also considered as DM, there tended to be differences in the HDR IBT ratio $(23.4 \% v s .8 .7 \% ; p=0.100)$. The reason why active use of HDR IBT influences DM may be explained with respect to cancer and immunity. HDR IBT may induce cancer cell death more rapidly than conventional fractionated central pelvic EBRT through the precise delivery of high doses during a short interval (12). EQD2 calculated by a linear quadratic model might partially reflect the radiobiological effect of HDR IBT. However, HDR IBT might be more effective for relatively late responsive cancer cells (alpha/beta ratio of $<10.0 \mathrm{~Gy}$ at point $\mathrm{A}$ ) than central pelvic EBRT because not all cancer cells are early responsive (alpha/beta ratio of $10 \mathrm{~Gy}$ at point A). Late-responsive cancer cells would increase during central pelvic EBRT because the CPD favours the eradication of early-responsive 


\begin{tabular}{|c|c|c|c|c|c|c|}
\hline & \multicolumn{3}{|c|}{ OS } & \multicolumn{3}{|c|}{ PFS } \\
\hline & HR & $95 \% \mathrm{CI}$ & $p$-Value & HR & $95 \% \mathrm{CI}$ & $p$-Value \\
\hline \multicolumn{7}{|l|}{ Univariate factors $^{\mathrm{a}}$} \\
\hline Age at diagnosis ( $>65$ years) & 4.27 & $1.42-12.83$ & $0.010 *$ & 2.41 & $0.92-6.36$ & 0.074 \\
\hline Histology (ADSC/ADC) & 1.48 & $0.019-11.38$ & 0.704 & 1.06 & $0.14-7.92$ & 0.964 \\
\hline Pelvic lymph node involvement & 0.57 & $0.19-1.30$ & 0.153 & 0.62 & $0.24-1.64$ & 0.339 \\
\hline Haemoglobin level (>11 g/dl) & 0.44 & $0.15-1.27$ & 0.130 & 0.46 & $0.18-1.22$ & 0.119 \\
\hline SCC antigen level $(>4.4 \mathrm{ng} / \mathrm{ml})$ & 2.68 & $0.84-8.54$ & 0.096 & 2.78 & $0.98-7.90$ & 0.055 \\
\hline Overall treatment duration ( $>56$ days) & 0.83 & $0.23-3.01$ & 0.775 & 0.75 & $0.24-2.32$ & 0.620 \\
\hline Total dose (EQD2) (>70 Gy) & 1.48 & $0.52-4.23$ & 0.467 & 1.57 & $0.60-4.07$ & 0.358 \\
\hline Pre-treatment tumour volume (V1 >50 cc) & 1.96 & $0.63-6.09$ & 0.243 & 2.44 & $0.88-6.74$ & 0.085 \\
\hline Tumour asymmetry (anterior/posterior) & 0.56 & $0.15-2.05$ & 0.378 & 0.58 & $0.18-1.81$ & 0.347 \\
\hline HDR IBT ratio $(>0.43)$ & 0.17 & $0.04-0.63$ & $0.008^{*}$ & 0.23 & $0.07-0.073$ & $0.013^{*}$ \\
\hline \multicolumn{7}{|l|}{ Multivariate factors } \\
\hline Age at diagnosis ( $>65$ years) & 3.09 & $0.99-9.62$ & 0.052 & 2.23 & $0.74-6.64$ & 0.152 \\
\hline SCC antigen level $(>4.4 \mathrm{ng} / \mathrm{ml})$ & 2.04 & $0.62-6.67$ & 0.240 & 2.55 & $0.80-8.08$ & 0.111 \\
\hline Pre-treatment tumour volume (V1 >50 cc) & - & - & - & 2.62 & $0.90-7.59$ & 0.076 \\
\hline HDR IBT ratio $(>0.43)$ & 0.26 & $0.06-1.02$ & 0.053 & 0.25 & $0.06-0.96$ & $0.043^{*}$ \\
\hline
\end{tabular}

ADSC, Adenosquamous carcinoma; ADC, adenocarcinoma; CI, confidence interval; EQD2, equivalent dose in 2 Gy fractions using an alpha/beta ratio of 10 Gy at point A; HDR, high-dose rate; HR, hazard ratio; IBT, intracavitary brachytherapy; OS, overall survival; PFS, progression-free survival; SCC, squamous cell carcinoma. aFactors with a value of $p<0.1$ from the univariate analysis were included in the multivariate analysis. *Significantly at $p<0.05$.

cancer cells. Therefore, escalation of the HDR IBT dose (EQD2) might reduce the risk of DMs and local progression by reducing late-responsive cancer cells, which could be sustained if they were treated with central pelvic EBRT. In addition, recent studies have demonstrated that lymphopenia during central pelvic EBRT was associated with a poor response and shorter survival times in various types of cancer (13-16). These studies were supported by increasing evidence of a link between tumour-infiltrating lymphocytes and cancer prognosis (17). Central pelvic EBRT may reduce circulating lymphocytes, as well as cancer cells, and this may be related to a poor survival (18). In contrast, HDR IBT is more likely to sustain peripheral lymphocytes due to its physical properties and this could complement central pelvic EBRT-related lymphopenia. Furthermore, HDR IBT may increase tumour-specific immune responses as in the case of prostate cancer (19). Nevertheless, the systemic effect of HDR IBT in the treatment of cervical cancer is poorly understood and requires further investigation.

Our findings demonstrate that an important factor in reducing late complications is increased HDR IBT doses (EQD2) relative to the total dose (EQD2). All late complications requiring intervention occurred in patients with low relative HDR IBT doses (EQD2). These patients were treated with a total dose of $\geq 75.0$ Gy (EQD2), including a CPD of $>44.0$ Gy (EQD2). This was consistent with previously published studies in which patients with FIGO stage IIIB cervical cancer treated with a CPD of 40.0-45.0 Gy (EQD2) and high relative low-dose-rate IBT had the lowest complication rates $(2,20)$, considering the similarity between low-dose-rate IBT and HDR IBT in the development of severe complications. We might infer that irradiation of a large volume of the rectum and bladder by EBRT could be more significant in treating morbidity than the irradiation of a small volume of the pericervical organ by HDR IBT.

Consequently, it may be advantageous for survival to administer HDR IBT as much as possible instead of central pelvic EBRT for primary tumour control. According to this result, we might consider HDR IBT at 3-4 weeks to increase the HDR IBT ratio if the dose coverage for HDR IBT of the primary tumour was retained despite a poor response to central pelvic EBRT. In this case, MRI-guided HDR IBT may help to increase the HDR IBT ratio (21).

Although, as far as we are aware, this study is the first to demonstrate the clinical significance of the HDR IBT ratio in FIGO stage IIB cervical cancer, there are several limitations. Firstly, we were unable to establish whether a wide range of HDR IBT ratios would aid in improving clinical outcomes, because our study did not include HDR IBT ratios $>0.48$. Secondly, this was a retrospective study of a relatively small number of patients. Therefore, future prospective studies are warranted. Despite its limitations, this study has merit in analyzing a homogeneous group of patients treated with full cycles of cisplatin-based CCRT 
followed by HDR IBT (EQD2) and in revealing that there are no significant differences in conventional prognostic factors between groups with high and low HDR IBT ratios.

In conclusion, a high HDR IBT ratio can improve DSS and PFS rates in patients with FIGO stage IIB cervical cancer. Increasing HDR IBT use in determining clinical total doses (EQD2) also reduces disease progression. Active administration of HDR IBT beyond previously accepted levels may be necessary for the treatment of locally advanced cervical cancer. Therefore, future prospective studies are warranted to further investigate this.

\section{References}

1 Rose PG, Bundy BN, Watkins EB, Thigpen JT, Deppe G, Maiman MA, Clarke-Pearson DL and Insalaco S: Concurrent cisplatin-based radiotherapy and chemotherapy for locally advanced cervical cancer. N Engl J Med 340: 1144-1153, 1999.

2 Logsdon MD and Eifel PJ: Figo IIIB squamous cell carcinoma of the cervix: an analysis of prognostic factors emphasizing the balance between external beam and intracavitary radiation therapy. Int J Radiat Oncol Biol Phys 43: 763-775, 1999.

3 Han K, Milosevic M, Fyles A, Pintilie M and Viswanathan AN: Trends in the utilization of brachytherapy in cervical cancer in the United States. Int J Radiat Oncol Biol Phys 87: 111-119, 2013.

4 Viswanathan AN, Creutzberg CL, Craighead P, McCormack M, Toita T, Narayan K, Reed N, Long H, Kim HJ, Marth C, Lindegaard JC, Cerrotta A, Small W Jr. and Trimble E: International brachytherapy practice patterns: a survey of the Gynecologic Cancer Intergroup (GCIG). Int J Radiat Oncol Biol Phys 82: 250-255, 2012.

5 Toita T, Kato S, Niibe Y, Ohno T, Kazumoto T, Kodaira T, Kataoka M, Shikama N, Kenjo M, Tokumaru S, Yamauchi C, Suzuki O, Sakurai H, Numasaki H, Teshima T, Oguchi M, Kagami Y, Nakano T, Hiraoka M and Mitsuhashi N: Prospective multi-institutional study of definitive radiotherapy with highdose-rate intracavitary brachytherapy in patients with nonbulky $(<4-\mathrm{cm})$ stage I and II uterine cervical cancer (JAROG0401/ JROSG04-2). Int J Radiat Oncol Biol Phys 82: e49-56, 2012.

6 Toita T, Kitagawa R, Hamano T, Umayahara K, Hirashima Y, Aoki Y, Oguchi M, Mikami M, Takizawa K and Cervical Cancer Committee of Japanese Gynecologic Oncology G: Phase II study of concurrent chemoradiotherapy with high-dose-rate intracavitary brachytherapy in patients with locally advanced uterine cervical cancer: efficacy and toxicity of a low cumulative radiation dose schedule. Gynecol Oncol 126: 211-216, 2012.

7 Chun M, Kang S, Ryu H, Chang K, Oh Y, Ju H and Lee E: Modified partial hyperfractionation in radiotherapy for bulky uterine cervical cancer: reduction of overall treatment time. Int J Radiat Oncol Biol Phys 47: 973-977, 2000.

8 Rose PG, Java J, Whitney CW, Stehman FB, Lanciano R, Thomas GM and DiSilvestro PA: Nomograms predicting progression-free survival, overall survival, and pelvic recurrence in locally advanced cervical cancer developed from an analysis of identifiable prognostic factors in patients from NRG Oncology/Gynecologic Oncology Group randomized trials of chemoradiotherapy. J Clin Oncol 33: 2136-2142, 2015.
9 An J-J, Lee Y-Y, Park JY, Choi CH, Kim T-J, Lee J-W, Kim B$\mathrm{G}$ and Bae D-S: Prognostic value of pre-treatment SCC-Ag level in patients with cervical cancer. Korean J Obstet Gynecol 54: 428-434, 2011

10 Girinsky T, Rey A, Roche B, Haie C, Gerbaulet A, Randrianarivello $\mathrm{H}$ and Chassagne D: Overall treatment time in advanced cervical carcinomas: a critical parameter in treatment outcome. Int J Radiat Oncol Biol Phys 27: 1051-1056, 1993.

11 Mitchell PA, Waggoner S, Rotmensch J and Mundt AJ: Cervical cancer in the elderly treated with radiation therapy. Gynecol Oncol 71: 291-298, 1998

12 Hall EJ and Giaccia AJ: Radiobiology for the radiologist. Philadelphia: Lippincott Williams \& Wilkins, 2006.

13 Lissoni P, Meregalli S, Bonetto E, Mancuso M, Brivio F, Colciago $\mathrm{M}$ and Gardani G: Radiotherapy-induced lymphocytopenia: changes in total lymphocyte count and in lymphocyte subpopulations under pelvic irradiation in gynecologic neoplasms. J Biol Regul Homeost Agents 19: 153-158, 2005.

14 Cho O, Oh YT, Chun M, Noh OK, Hoe JS and Kim H: Minimum absolute lymphocyte count during radiotherapy as a new prognostic factor for nasopharyngeal cancer. Head Neck 38(Suppl 1): E1061-1067, 2016.

15 Cho O, Oh YT, Chun M, Noh OK and Lee HW: Radiationrelated lymphopenia as a new prognostic factor in limited-stage small cell lung cancer. Tumour Biol 37: 971-978, 2016.

16 Heo J, Chun M, Noh OK, Oh YT, Suh KW, Park JE and Cho O: Sustaining blood lymphocyte count during preoperative chemoradiotherapy as a predictive marker for pathologic complete response in locally advanced rectal cancer. Cancer Res Treat 48: 232-239, 2016.

17 Gooden MJ, de Bock GH, Leffers N, Daemen T and Nijman HW: The prognostic influence of tumour-infiltrating lymphocytes in cancer: a systematic review with meta-analysis. Br J Cancer 105: 93-103, 2011

18 Cho O, Chun M, Chang SJ, Oh YT and Noh OK: Prognostic value of severe lymphopenia during pelvic concurrent chemoradiotherapy in cervical cancer. Anticancer Res 36: 35413547, 2016.

19 Nesslinger NJ, Sahota RA, Stone B, Johnson K, Chima N, King C, Rasmussen D, Bishop D, Rennie PS, Gleave M, Blood P, Pai $\mathrm{H}$, Ludgate $\mathrm{C}$ and Nelson BH: Standard treatments induce antigen-specific immune responses in prostate cancer. Clin Cancer Res 13: 1493-1502, 2007.

20 Patel FD, Sharma SC, Negi PS, Ghoshal S and Gupta BD: Low dose rate $v s$. high dose rate brachytherapy in the treatment of carcinoma of the uterine cervix: a clinical trial. Int $\mathbf{J}$ Radiat Oncol Biol Phys 28: 335-341, 1994.

21 Potter R, Dimopoulos J, Georg P, Lang S, Waldhausl C, Wachter-Gerstner N, Weitmann H, Reinthaller A, Knocke TH, Wachter S and Kirisits C: Clinical impact of MRI assisted dose volume adaptation and dose escalation in brachytherapy of locally advanced cervix cancer. Radiother Oncol 83: 148-155, 2007.

Received October 21, 2016

Revised November 15, 2016

Accepted November 23, 2016 